\title{
Enquête
}

Archives de la revue Enquête

3| 1996

Interpréter, Surinterpréter

\section{La « stratégie » chez Pierre Bourdieu}

Note de lecture

Strategy in Bourdieu's works. Reading note

\section{Alain Dewerpe}

\section{(2) OpenEdition}

Journals

Édition électronique

URL : http://journals.openedition.org/enquete/533

DOI : $10.4000 /$ enquete. 533

ISSN : 1953-809X

Éditeur :

Cercom, Éditions Parenthèses

\section{Édition imprimée}

Date de publication : 1 novembre 1996

Pagination : 191-208

\section{Référence électronique}

Alain Dewerpe, «La « stratégie » chez Pierre Bourdieu », Enquête [En ligne], 3 | 1996, mis en ligne le 11 juillet 2013, consulté le 10 décembre 2020. URL : http://journals.openedition.org/enquete/533 ; DOI : https://doi.org/10.4000/enquete.533

Ce document a été généré automatiquement le 10 décembre 2020. 


\title{
La «stratégie » chez Pierre Bourdieu
}

\author{
Note de lecture \\ Strategy in Bourdieu's works. Reading note
}

\section{Alain Dewerpe}

1 Qu'est-ce qu'une stratégie selon Pierre Bourdieu? Certainement un système d'idées complexe et cohérent, d'autant plus difficile à saisir que le concept est utilisé sur une longue période de recherche - plus de vingt ans - et qu'il faut prendre en compte les tâtonnements, essais et erreurs, hypothèses abandonnées qui forment, dans le déroulement des textes mêmes, l'histoire d'un concept au travers de son usage pratique. D'autant plus difficile à saisir, ensuite, parce qu'il s'intègre dans un système de notions connexes, tant et si bien qu'expliciter l'une revient à reconstruire le système dans son entier. D'autant plus difficile, enfin, que la logique agonistique de laquelle procède le choix intellectuel et sémantique des notions est stratégiquement (au sens premier du terme) construite pour s'opposer, en particulier par l'emprunt à d'autres sciences sociales - économie et anthropologie -, à d'autres notions adverses dans le champ de la sociologie ${ }^{1}$

2 Comme celui de capital, le sens de stratégie est très élargi² ${ }^{2}$, bien au-delà, à lire le Robert, du sens militaire premier - la partie de la science militaire qui concerne la conduite générale de la guerre et l'organisation de la défense d'un pays -, ou du sens induit aux registres non guerriers, un ensemble d'actions coordonnées, de manœuvres, en vue d'une victoire.

«Les stratégies de reproduction, ensemble de pratiques phénoménalement très différentes par lesquelles les individus ou les familles tendent, inconsciemment et consciemment, à conserver ou à augmenter leur patrimoine et, corrélativement, à maintenir ou améliorer leur position dans la structure des rapports de classe, constituent un système qui, étant le produit d'un même principe unificateur et générateur, fonctionne et se transforme en tant que tel. Par l'intermédiaire de la disposition à l'égard de l'avenir, elle-même déterminée par les chances objectives de reproduction du groupe, ces stratégies dépendent premièrement du volume et de la structure du capital à reproduire, c'est-à-dire du volume actuel et potentiel du capital économique, du capital culturel et du capital social possédés par le groupe et 
leur poids relatif dans la structure patrimoniale ; et deuxièmement de l'état, luimême fonction de l'état du rapport de force entre les classes, du système des instruments de reproduction, institutionnalisés ou non ${ }^{3}$."

Soit une séquence logique qui peut se représenter par le graphique simplifié suivant :

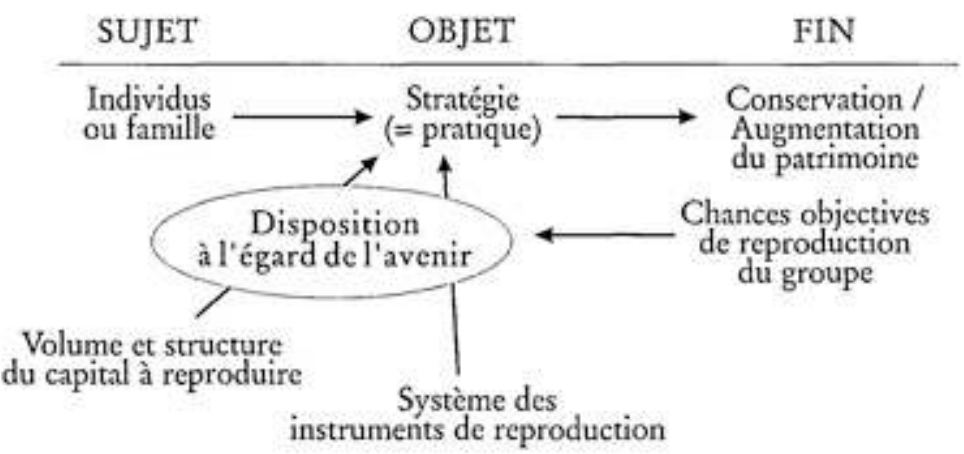

3 Disposant d'un certain capital, dans un champ structuré par des règles et des enjeux, un agent adopte une stratégie pour en tirer le maximum de profit. Non point moyen de l'action, manœuvre ou plan, la stratégie apparaît comme un modèle de comportement, un mode d'action ou de conduite, une pratique qui a des effets bénéfiques, voire optimaux, conçus en terme de "profit », bref un acte finalisé : c'est ce qu'on fait dans le monde social présent dans sa relation avec le monde social futur, compte tenu du monde social passé. S'appuyant sur un intérêt subjectif comme sur un intérêt objectif, opérant à court comme à long terme, cette notion polysémique est par définition, d'une part, diversifiée : «Une structure déterminée de capital tend à imposer un mode de reproduction particulier, caractérisé par un ensemble de stratégies de reproduction adapté aux particularités de l'espèce de capital qu'il s'agit de reproduire ${ }^{4} »$; et, d'autre part, systématique : il y a un "système des stratégies de reproduction [...] constitutif d'un mode de reproduction ».

4 Cette stratégie n'a pas besoin d'être délibérément choisie, elle peut être d'autant plus efficace qu'elle n'est pas intentionnelle. Des «pratiques phénoménalement très différentes s'organisent objectivement », elles contribuent à la reproduction du capital possédé : mais ce raisonnement ne conduit pas à « imputer au calcul rationnel, ou même à l'intention stratégique » ces pratiques ${ }^{5}$.

«L'habitus est au principe d'enchaînement de "coups" qui sont objectivement organisés comme des stratégies sans être aucunement le produit d'une véritable intention stratégique (ce qui supposerait par exemple qu'ils soient appréhendés comme une stratégie parmi d'autres possibles) ${ }^{6}$. »

«Je dois insister une fois encore sur le fait que le principe des stratégies philosophiques (ou littéraires, etc.) n'est pas le calcul cynique, la recherche consciente de la maximisation d'un profit spécifique, mais une relation inconsciente entre un habitus et un champ. Les stratégies dont je parle sont des actions objectivement orientées par rapport à des fins qui peuvent n'être pas les fins subjectivement poursuivies ${ }^{7}$. "

5 En effet, cette organisation objective vient de ce que les pratiques ont pour principe l'habitus, «qui tend à reproduire les conditions de sa propre production en produisant, dans les domaines les plus différents de la pratique, les stratégies objectivement cohérentes et caractéristiques d'un mode de reproduction ${ }^{8}$ ». "Système de dispositions acquises par l'apprentissage implicite ou explicite qui fonctionne comme un système de schèmes générateurs ", l'habitus est "générateur de stratégies ${ }^{9}$ ", valant pour des 
situations répétées mais aussi dans des conjonctures nouvelles, où il joue «en tant que principe générateur de stratégies permettant de faire face à des situations imprévues ».

6 Le concept, absent dans les années soixante, s'impose avec l'Esquisse d'une théorie de la pratique, puis s'intègre dans le système théorique élaboré à la fin des années soixante-dix. En dépouillant les index, on constate son absence ${ }^{10}$, puis sa présence : stratégie renvoie alors à habitus, reproduction, rite ${ }^{11}$; à règles ${ }^{12}$; à calcul, rhétorique, système de défense collectif ${ }^{13}$. Par ailleurs, la stratégie s'exprime au singulier ${ }^{14}$, mais aussi au pluriel ${ }^{15}$. Si l'on envisage ses spécifications, on constate que la stratégie peut être collective, d'honneur, de fécondité, matrimoniale, successorale ${ }^{16}$; ou : de fécondité, de reproduction, d'officialisation ${ }^{17}$. Au pluriel, elles peuvent être individuelles, collectives, à plusieurs générations, économiques, symboliques, de bluff, de condescendance, de contre-pied et de double négation, de distinction, d'euphémisation, de reconversion, de reproduction, de fécondité, de scolarisation ${ }^{18}$; ou : économiques, d'investissement social, de reproduction, de sociodicée, éducatives ou scolaires, de fécondité, matrimoniales, prophylactiques, successorales ${ }^{19}$. L'extension est (trop ?) large.

7 En un sens, la stratégie a affaire à la continuité intergénérationnelle, aux instances individuellement les moins consciemment (en apparence ?) contrôlables - mariages, procréation, scolarisation -, celles où la transparence du marché est la moins saisissable c'est-à-dire à la mobilité sociale du groupe restreint (la famille surtout) et à la reproduction, éventuellement élargie, des ressources sociales de ces groupes.

«Indissociables des stratégies successorales, des stratégies de fécondité, ou même des stratégies pédagogiques, c'est-à-dire de l'ensemble des stratégies de reproduction biologique, culturelle et sociale que tout groupe met en œuvre pour transmettre à la génération suivante, maintenus ou augmentés, les pouvoirs et les privilèges hérités, les stratégies matrimoniales n'ont pour principe ni la raison calculatrice ni les déterminations mécaniques de la nécessité économique, mais les dispositions inculquées par les conditions d'existence, sorte d'instinct socialement constitué qui porte à vivre comme nécessité inéluctable du devoir ou comme appel irrésistible du sentiment les exigences objectivement calculables d'une forme particulière d'économie ${ }^{20}$. »

8 Tout est ici d'abord affaire de persévérance dans son état et, pour ceux qui ont des ressources plus que pour ceux qui n'en ont pas, de pouvoirs. Filant la métaphore du monde social comme jeu, la stratégie a ainsi affaire avec le sens du jeu, les règles, les «coups» ("l'image du jeu est sans doute la moins mauvaise pour évoquer les choses sociales ") $)^{21}$, mais un sens dont personne n'a jamais fixé les règles, alors que le jeu luimême est soumis à des régularités qui en assurent la cohérence. S'inscrivant dans un système théorique, organisée en étroite relation avec les concepts connexes d'habitus et de capital, la stratégie est néanmoins l'objet de manifestes limites, théoriques elles aussi, qui sont explicitement assumées: "C'est [...] un terme que je n'emploie pas sans hésitation ».

« [Le terme de stratégie] encourage le paralogisme fondamental, celui qui consiste à donner le modèle qui rend raison de la réalité pour constitutif de la réalité décrite, en oubliant le "tout se passe comme si", qui définit le statut propre du discours théorique. Plus précisément, il incline à une conception naïvement finaliste de la pratique (celle qui soutient l'usage ordinaire de notions comme intérêt, calcul rationnel, etc.). En fait, tout mon effort vise au contraire, avec la notion d'habitus par exemple, à rendre compte du fait que les conduites (économiques ou autres) prennent la forme de séquences objectivement orientées par référence à une fin, sans être nécessairement le produit, ni d'une stratégie consciente, ni d'une détermination mécanique ${ }^{22}$.» 
On s'interdit donc d'en rendre pleinement compte si l'on n'introduit pas dans le commentaire l'avantage pratique de son usage, qui découle de la position que la notion occupe dans le dispositif des refus théoriques de l'auteur. La stratégie se pose ainsi contre l'économisme, qui envisage, d'un côté, de par sa composante subjectiviste, un acteur rationnel orientant son action vers la recherche de l'optimum et une fin consciemment posée, et envisage, de l'autre côté, de par sa composante objectiviste, des causes économiques mécaniques: bref elle se conçoit à la fois comme une arme contre le finalisme et contre le mécanisme. On comprendra ainsi mieux le sens des limites, voire des apories, du concept de stratégie, si l'on prend en compte le programme de double refus du subjectivisme sartrien (doublon philosophique de l'économisme de l'acteur rationnel) et de l'objectivisme structuraliste ${ }^{23}$. La stratégie est alors dans une position paradoxale, à la fois stratégique parce qu'elle monte en première ligne contre ses deux adversaires opposés mais alliés, et précaire parce qu'elle est ainsi soumise à leurs feux croisés.

«Il y a une économie des pratiques, c'est-à-dire une raison immanente aux pratiques, qui ne trouve son "origine" ni dans les "décisions" de la raison comme calcul conscient, ni dans les déterminations de mécanismes extérieurs et supérieurs aux agents. [...], faute de reconnaître aucune autre forme d'action que l'action rationnelle ou la réaction mécanique, on s'interdit de comprendre la logique de toutes les actions qui sont raisonnables sans être le produit d'un dessein raisonné ou, à plus forte raison, d'un calcul rationnel; habitées par une sorte de finalité objective sans être consciemment organisées par rapport à une fin explicitement constituée ; intelligibles et cohérentes sans être issues d'une intention de cohérence et d'une décision délibérée; ajustées au futur sans être le produit d'un projet ou d'un plan ${ }^{24}$."

10 De ce programme du ni-ni - ni raison calculatrice, ni déterminations mécaniques de la nécessité économique, ni stratégie consciente, ni détermination automatique - découle le fait que la stratégie de Pierre Bourdieu est un concept en creux, une notion en suspens, comme sur le fil du rasoir. Plusieurs questions me paraissent en effet faire problème, dans l'écart existant entre la logique théorique, close dans une très grande cohérence, et l'usage pratique, à partir des terrains d'enquête effectivement prospectés: elles ressortissent à la part de l'historicité, aux échelles de décision et à la confusion des logiques d'un niveau à l'autre, aux balancements entre les conditions de la décision individuelle et celles de la décision collective, entre le conscient et l'inconscient, entre la logique de la détermination et celle de l'invention.

\section{Un historicisme statique}

11 La stratégie est un concept totalement historicisé. En ce que seule l'histoire des champs peut rendre raison de sa structure et de sa répartition: "À travers la connaissance pratique des principes du jeu qui est tacitement exigée des nouveaux entrants, c'est toute l'histoire du jeu, tout le passé du jeu, qui sont présents dans chaque acte du jeu ${ }^{25} »$. Ainsi l'histoire est présente en ce qu'elle est incorporée dans un état présent. Mais l'est-elle comme dynamique? La relation entre les modes de production et les modes de reproduction paraît peu claire. Comment rendre en effet raison, pratiquement, de la dynamique du changement et de la continuité ? Comment passer d'un mode de reproduction à un autre? S'il y a des stratégies les plus probables, compte tenu des habitus des agents, comment se réalise la rupture, le passage à une stratégie intrinsèquement nouvelle? En d'autres termes, on voit bien que tout est censé changer, 
mais on comprend mal comment. On conçoit que ce nouveau soit empiriquement rare : mais, par exemple, pour l'historien de la "révolution industrielle ", l'approche de cette nouveauté (le système de la concentration du procès de travail et de la mécanisation) sera décisive.

Or, il n'y a paradoxalement que peu d'historicité dans la logique de P. Bourdieu. Trois arguments vont dans ce sens. D'abord, les champs sont conçus comme des plans factoriels, de telle sorte que les règles de transformation d'un plan à l'autre sont soit absentes, soit sous-estimées dans l'analyse, ce qui mène de facto à l'absence d'historicité26 : «On peut décrire le champ social comme un espace multidimensionnel de positions tel que toute position actuelle peut être définie en fonction d'un système multidimensionnel de coordonnées ${ }^{27}$ ». À la question: "Vous décrivez un état de la structure sociale sans dire comment ça change », P. Bourdieu précise :

«Ce que saisit l'enquête statistique, c'est un moment, un état du jeu à 2, 3, 4 ou 6 joueurs, n'importe; elle donne une photographie des piles de jetons de différentes couleurs qu'ils ont gagnés lors des coups précédents et qu'ils vont engager dans les coups suivants. Le capital saisi dans l'instant est un produit de l'histoire qui va produire de l'histoire ${ }^{28}$."

13 Ici, la métaphore du jeu (quelque chose entre le bridge et le Monopoly) n'éclaire pas vraiment le fait qu'avec une même donne, dans un état du champ semblable, deux joueurs auront des stratégies différentes et qu'en particulier, dans le cas de stratégies improbables, l'un aura une stratégie innovante et l'autre pas. Ensuite, et je suis ici $\mathrm{J}$. Rancière, le système reproduit son existence parce qu'il est méconnu et sa reproduction produit la méconnaissance :

«Il est impossible d'imaginer que l'ordre puisse jamais cesser de contribuer "par son existence même" à sa "propre perpétuation"; impossible donc qu'il ne (re)produise pas perpétuellement ce surcroît de la méconnaissance appelé à se redoubler en méconnaissance de la méconnaissance et ainsi de suite à l'infini ${ }^{29}$ ".

Enfin, à la limite, l'histoire est improbable (impossible ?) au sens où ceux qui la font sont des agents à la trajectoire improbable :

«Il ne faut pas oublier tous les décalages entre l'histoire incorporée et l'histoire réifiée, tous les gens qui sont "mal dans leur peau" [...], c'est-à-dire dans leur poste, dans la fonction qui leur est assignée. Ces gens en porte-à-faux, déclassés par le bas ou par le haut, sont des gens à histoires qui, souvent, font l'histoire ${ }^{30}$. "

Si bien qu'il y a de l'histoire partout, en ce que les stratégies possibles ne sont que les effets par lesquels l'histoire passée a structuré l'état présent du champ où elles s'exercent, et qu'il n'y a d'histoire nulle part, puisque la dynamique de l'invention paraît réduite au minimum du « sursis », suivant l'aphorisme selon lequel il faut bien que tout change pour que tout demeure pareil.

\section{Le stratège, entre moi et les autres}

L'acteur de la stratégie pose un second problème, dès lors qu'il est un agent socialisé. Cette dernière formule ne nous aide pas beaucoup si elle veut seulement dire que tout agent s'inscrit et inscrit son action dans le monde social. Mais elle veut dire plus : elle suggère une réduction de l'initiative de l'agent, le refus de l'individualisme méthodologique comme glissement du phénomène social aux choix et calculs d'individus supposés dotés d'une rationalité limitée mais suffisante. La distinction entre décision individuelle et décision collective est faite, souvent implicitement déductible des 
exemples, mais jamais explicitée. Parti de l'individu ou de la famille, on passe au collectif par agrégation, avec des effets de composition, dits "pervers", ce qui mène à la dénonciation de l'illusion biographique comme censure de la «trajectoire comme série des positions successivement occupées par un même agent (ou même un groupe) dans un espace lui-même en devenir et soumis à d'incessantes transformations ${ }^{31} »$ : «On voit que le sujet n'est pas l'ego instantané d'une sorte de cogito singulier, mais la trace individuelle de toute une histoire collective. [...] En fait, on ne sait plus qui est le sujet de la décision finale ${ }^{32}$.»

17 Cette dilution suggère que les règles de construction sociale des placements et des déplacements dans l'espace social seraient de même ordre, réductibles à une même espèce, ou, du moins, que l'on pourrait tenir pour négligeable une interrogation sur les variations, les écarts, les ruptures, de l'une à l'autre, comme nous le suggèrent les problèmes posés par l'écriture de la biographie historique ${ }^{33}$. Il faut ainsi préjuger ou construire l'homologie de la décision individuelle et des choix collectifs. Mais, comment, par exemple, s'articulent la décision industrialiste de l'entrepreneur et les voies sociales, collectives, nationales de l'industrialisation ${ }^{34}$ ? On voit bien que la stratégie paternaliste de Motte-Bossut ou des Schneider n'est pas du même ordre que les stratégies, collectives ou impersonnelles (affectées à des instances globales, - le "patronat », le «capital »certes réifiantes, mais dont l'historien a souvent besoin pour rendre compte de phénomènes sociaux globaux) de différenciation sociale de la force de travail, par les procédures de segmentation et différenciation du marché du travail et du procès de travail. Ou : quelque chose comme la division internationale du travail est-elle le produit d'une stratégie?

\section{Science de la stratégie et inconscient social}

La conscience de soi dans l'action produite par la stratégie constitue le troisième problème. On voit bien chez Bourdieu la dialectique de l'objectivité et de la subjectivité chez l'agent individuel. Mais on constate aussi une double modalité de l'action et de la stratégie, consciente et inconsciente, avec un glissement entre méconnaissance (freudienne) et dénégation (sartrienne) ${ }^{35}$. D'où une série de questions. Est-ce que je sais que je suis un stratège? Est-ce que je sais quelle stratégie j'ai adoptée? Est-ce que la stratégie adoptée est conforme à l'idée consciente que j'ai de mes intérêts ? Est-ce que la stratégie que j'ai adoptée est conforme à mes intérêts objectifs ? Est-ce que la stratégie mise en œuvre a été efficace? P. Bourdieu met tout particulièrement l'accent sur le caractère non conscient des objectifs poursuivis et les effets de méconnaissance qui en sont la conséquence (le désintéressement), jusqu'à faire de cette dissimulation une finalité fonctionnelle de l'efficacité de la stratégie. À propos du don et du contre-don, il note: «Tout se passe comme si les stratégies, et en particulier celles qui consistent à jouer avec le tempo de l'action ou, dans l'interaction, avec l'intervalle entre les actions, s'organisaient en vue de dissimuler, à soi et aux autres, la vérité de la pratique ${ }^{36}$. »

Les «stratégies les plus "payantes" sont celles qui ne se vivent pas comme des stratégies ${ }^{37}$ ». La primauté semble donc être du côté de l'inconscient, si l'on se réfère à la double proposition selon laquelle le système reproduit son existence parce qu'il est méconnu, et que le système opère, par la reproduction de son existence, un effet de méconnaissance. De fait, la stratégie semble réaliser cette magie sociale qui consiste à masquer le monde social, à produire un effet de méconnaissance, effet qui est au principe de l'action 
efficace, c'est-à-dire porteuse d'un accroissement du profit de l'agent, et dont la vérité serait la stratégie d'officialisation, qui a pour objectif de transformer en le masquant l'intérêt en désintéressement ${ }^{38}$.

20 Mais le mécanisme qui déclenche les stratégies non conscientes n'est pas explicitée ${ }^{39}$, si bien que l'ambiguïté entre stratégie consciente et inconsciente donne du poids à l'imputation, caricaturale et grossissant le trait, d'une interprétation par une conspiration mythique de la classe dominante ${ }^{40}$.

Or, nous avons besoin de connaître avec précision le registre du conscient, du stratégique stricto sensu, et celui de l'inconscient, les règles qui régissent la frontière entre l'un et l'autre - faute de quoi, nous échapperont les logiques de transformation de l'expérience du monde social qu'ont les agents - afin d'obéir ainsi à l'injonction que Bourdieu adresse lui-même au savant :

«La théorie la plus résolument objectiviste doit intégrer la représentation que les agents se font du monde social et, plus précisément, la contribution qu'ils apportent à la construction de la vision de ce monde, et, par là, à la construction même de ce monde, à travers le travail de représentation (à tous les sens du terme) qu'ils ne cessent d'accomplir pour imposer leur vision du monde ou la vision de leur propre position dans ce monde, de leur identité sociale ${ }^{41}$. »

Car c'est bien à partir de cette représentation du monde actuel que se construisent, pour l'individu et pour les groupes, les représentations du monde possible et souhaitable qui sont au principe des stratégies conscientes que ces individus ou ces groupes vont mettre en œuvre: le sens du jeu est ainsi en partie déterminé par des instances telles que la quantité d'informations disponibles à chaque agent. La question est d'autant plus cruciale qu'on sait que les théories de la décision distinguent, certes abstraitement, la décision en univers certain, aléatoire (probabilités connues), et indéterminé, avec leurs variantes de critères (maximin, minimax, maximax, matrice des regrets, etc.). Un exemple: un entrepreneur (ou le groupe des entrepreneurs), ayant lu Charles Babbage et Andrew Ure et ayant été convaincu par eux des avantages de la division du travail pour l'accumulation du capital (elle induit l'annexion d'un travailleur à une seule opération, ce qui augmente la productivité, assure le monopole du contrôle sur le procès de travail, l'achat de la force de travail est au niveau de chacune des opérations décomposées et non à celui de la qualification la plus élevée), est-il conscient de et formule-t-il une stratégie de mécanisation / division du travail ? ${ }^{42}$

\section{Les fins de la conduite : un intérêt inintéressant}

La logique constitutive de l'action fait elle-même problème. Les principes qui sont censés fixer les buts - conscients ou inconscients - de l'action relèvent d'une catégorie hétérogène, l'intérêt :

«Il n'y a pas un intérêt, mais des intérêts, variables selon les temps et selon les lieux, à peu près à l'infini. Dans mon langage, je dirai qu'il y a autant d'intérêts qu'il $\mathrm{y}$ a de champs [...]. L'existence d'un champ spécialisé et relativement autonome est corrélative de l'existence d'enjeux et d'intérêts spécifiques ${ }^{43}$."

En théorie, contrairement à la réification de l'axiomatique de l'économie néo-classique, l'intérêt n'est pas donné une fois pour toutes, doit être spécifié historiquement, est une « institution arbitraire », une « raison d'être » de l'action ${ }^{44}$.

«Le mot d'intérêt, que j'ai plusieurs fois employé, est aussi très dangereux, parce qu'il risque d'évoquer un utilitarisme qui est le degré zéro de la sociologie. Cela dit, 
la sociologie ne peut se passer de l'axiome de l'intérêt, entendu comme l'investissement spécifique dans les enjeux, qui est à la fois la condition et le produit de l'appartenance à un champ ${ }^{45}$. "

"Autrement dit, l'intérêt est à la fois condition du fonctionnement d'un champ (champ scientifique, champ de la haute couture, etc.), en tant qu'il est ce qui "fait courir les gens", ce qui les fait concourir, se concurrencer, lutter, et produit du fonctionnement du champ ${ }^{46}$ ».

On peut signaler ici des difficultés de trois ordres :

1. Préoccupé de concevoir contre la transcendance du subjectivisme utilitariste, qu'illustre la Rational Action Theory (RAT) ${ }^{47}$, une théorie de l'action immanente, Bourdieu serait dans l'obligation de construire une « raison d'être » tout aussi essentialiste : tout se passe en pratique comme si une forme plus complexe de principe premier transcendant revenait, malgré toutes les précautions de protocole et de méthode, par la coulisse, dans la stratégie objective telle qu'il la pratique. Il y a une rationalité de l'agent, mais cette rationalité doit être pensée comme méconnaissance de soi : il faut imaginer qu'un agent et l'agent et la collectivité sur lesquels s'exerce son action, comme résultante de sa stratégie - puisse (et doive) ignorer qu'il exerce par là même une «imposture bien fondée, un abus de pouvoir légitime, collectivement méconnu, donc reconnu ${ }^{48} »$. Or, l'attention portée aux effets de méconnaissance induits par la distribution initiale des capitaux et à la structure du jeu et des enjeux instruit pratiquement une diminutio capitis de la part spécifique prise par la subjectivité de l'agent, son pouvoir d'invention, la diversité et la singularité de la rationalité qu'il met en œuvre, bref par ses «raisons d'être » (et d'agir). Les formes réelles des divers intérêts tendent à s'estomper.

2. Ensuite, «les préférences ne peuvent pas en même temps être engendrées par la nécessité et expliquées par leur fonction »: il y aurait deux directions contradictoires, expliquer les causes des choix par leurs conditions sociales de production et les fonctions par leurs effets sociaux ${ }^{49}$.

28 3. Enfin, il ne suffirait pas, dans une approche cynique, d'expliciter les attendus intéressés du désintéressement si l'on ne peut rendre raison d'une part, que les stratégies de désintéressement sont mises en œuvre par certains groupes plutôt que par d'autres, par exemple chez les serviteurs de l'État ou les professions libérales, et non dans le monde des affaires; d'autre part, que ces stratégies peuvent être faiblement rémunératrices, qu'on peut ne pas avoir intérêt au désintéressement (le jeune avocat stagiaire, le médecin sans cabinet ni clientèle, l'oblat, le pauvre prêtre, le cadet de famille, l'instituteur, qui « font les frais » de leur désintéressement); enfin qu'elles puissent s'appliquer aux déviants et marginaux, ceux qui n'ont pas le sens du jeu, jouent hors-champ, hors-norme, à contretemps ${ }^{50}$. En d'autres termes, une même stratégie peut relever de positions différentes - dominées ou dominantes, présentes ou passées, dans le jeu et hors du jeu - si bien que cette hétérogénéité fait douter de la logique de la pratique (maximiser un profit en masquant que c'est un profit) que la notion était censée éclairer ${ }^{51}$.

\section{L'économique généralisée}

La stratégie joue sur une métaphore de l'échange marchand, du capital, de l'économie: cette extension au discours sociologique du paradigme économique est explicitement assumée. «L'homo sociologicus est donc un joueur intéressé (le jeu comporte un ou des enjeux) et susceptible de calculer et de définir une stratégie approximativement 
rationnelle » : cette axiomatique de l'intérêt est une dérive directe de L'homo economicus ${ }^{52}$. Mais, contre l'usage naturalisant des «motivations » et des « aspirations » en sociologie, Bourdieu emprunte à l'économie un "intérêt», associé à «investissement» ou à «illusion ${ }^{53}$ ». Il s'agit d'une extension du lexique dans une nouvelle axiomatique qui fonderait une économique généralisée (une "économie générale des pratiques ») empruntant, en les transformant, des notions à l'économique de l'économie. Trois remarques:

1. Moins paradoxalement qu'il n'apparait à première vue, on trouve peu d'analyses du champ économique dans l'œuvre de P. Bourdieu, dont l'essentiel des études de cas ressortissent à ce qu'il est convenu d'appeler la sociologie de la culture ${ }^{54}$. Comment alors que la tendance est plutôt de dépasser la théorie néo-classique du marché et de L' homo economicus rationnel dans un univers transparent, de K. Polanyi ${ }^{55}$, ou d'A. Hirschman ${ }^{56}$, jusqu'à l'économie des conventions ${ }^{57}$ - se passe le retour de ces notions dans la sphère de l'économie d'où elles sont parties?

31 2. La mesure, affirmée théoriquement, est souvent pratiquement inexistante : dans le cas de la quantification des capitaux ${ }^{58}$; dans celui de la probabilité des trajectoires de l'écart entre position initiale, position probable d'arrivée, position réelle d'arrivée; dans celui de l'utilisation de stratégies homologues assurant l'accès à cette position d'arrivée (ne peuton envisager le cas d'une position d'arrivée probable obtenue grâce à une stratégie improbable?).

3. L'ambiguïté demeure en ce qu'en un sens « l'illusion du calcul économique universel » a peut-être « un fondement dans la réalité ». Puisque tous les champs ont une économie, même si elle leur est spécifique, ils peuvent « obéir, en partie ou en totalité, au principe d'économie et faire intervenir une forme de calcul, de ratio, visant à assurer l'optimisation du bilan coûts-profits ». Ces actions, dans des champs autres que l'économie restreinte, pourraient ainsi être interprétées comme «des investissements orientés vers la maximisation de l'utilité ». Mais, en un autre sens, cette universalisation de la rationalité économique de l'économique oublie que la « rationalisation parfaite n'est jamais réalisée ${ }^{59}$ ». Ce glissement et cette tension signalent une hésitation entre les bénéfices pratiques de l'économique généralisée, où l'on peut objectiver les rationalités spécifiques des différents champs en terme de calcul d'optimum, et les coûts théoriques d'une soumission absolue à la logique de l'intérêt économique des économistes.

\section{Sur le fil du rasoir}

33 À l'issue de cette brève approche des actions situées sur le terrain, les feux croisés de l'objectivisme et du subjectivisme n'ont pas été réduits.

Dans une analyse des rapports entre ethnologie et histoire, C. Lévi-Strauss s'interrogeait, à partir du cas des sociétés dites « à maisons » :

« N’y a-t-il pas contradiction à parler de structure là où je n'ai décrit qu'un jeu de rivalités entre des stratégies individuelles ou collectives? Plus précisément, ce que nous prenons pour une structure sociale d'un type particulier ne se réduit-il pas à une moyenne statistique résultant de choix faits en toute liberté, ou échappant $d u$ moins à toute détermination externe ? Comme il est peu plausible que les sociétés humaines se répartissent en deux groupes irréductibles, certaines relevant de la structure, d'autres de l'événement, douter que l'analyse structurale s'applique à quelques-unes conduit à les récuser pour toutes ${ }^{60}$. » 
Cette critique du "spontanéisme » et du « subjectivisme » (qui «traîne un peu partout » et qui est «à la mode »), que Bourdieu prend pour lui ${ }^{61}$, peut être inversée. Quel peut être le rôle de la stratégie, puisque le champ des possibles ouvert à l'« agent socialisé ", contre le "sujet", est déterminant et puisque les stratégies du sens pratique, même pensées, contre les projets et les calculs d'une conscience, en termes de probabilités statistiques, sont « plus ou moins "automatiques"62 " ? Il me semble que l'usage fait de la stratégie n'est parfois pas très éloigné de la conception de Durkheim suivant laquelle,

"si rien n'entrave ou ne favorise indûment les concurrents qui se disputent les tâches, il est inévitable que ceux-là seuls qui sont les plus aptes à chaque genre d'activités y parviennent. [...]. Ainsi se réalise de soi-même l'harmonie entre la constitution de chaque individu et sa condition ${ }^{63}$. »

Si on lit habitus au lieu d'aptitude, homologie au lieu d'harmonie, la logique de la relation entre l'action stratégiquement construite et l'univers des positions historiquement possibles est chez Bourdieu d'un ordre voisin. Si l'on accepte cette vue, le véritable choix créateur, celui qui fabrique, celui qui invente, voire celui qui tranche du probable vers l'improbable, n'a guère de place ici. Espace de liberté dans un monde sans liberté, univers singulier de l'art du jeu dans un monde où tout (donc rien) n'est jeu, la stratégie n'est peut-être plus que l'art de faire de nécessité, vertu.

À l'autre extrême du dispositif, la pluralité des possibles et des choix est maintenue en trois acceptions. D'abord, l'existence de dérives et de marges - jamais niées en théorie mais rarement mesurées en pratique - est interprétée comme écart à une valeur modale, à la régularité statistique affectant les dispositions déterminantes de la stratégie. Ensuite, le choix, dans la stratégie même, est ouvert parce qu'il demeure du "flou», de «l'indéterminé », du «suspens » et du «sursis ». Ainsi, des représentations du monde social, on dira que :

«Les objets du monde social peuvent être perçus de différentes façons parce que, comme les objets du monde naturel, ils comportent toujours une part d'indétermination et de flou - du fait par exemple que les combinaisons les plus constantes de propriétés ne sont jamais fondées que sur des liaisons statistiques entre des traits substituables; et aussi parce que, en tant qu'objets historiques, ils sont sujets à des variations dans le temps et que leur signification, dans la mesure où elle est suspendue à l'avenir, est elle-même en suspens, en attente, en sursis, et, par là, relativement indéterminée ${ }^{64}$.

Enfin, la stratégie comme produit du sens pratique comme sens du jeu est un pouvoir d'adaptation aux situations mouvantes, avec son potentiel d'erreur ou d'inadaptation (erreur de jeu, médiocre sens du jeu) :

«Le bon joueur, qui est en quelque sorte le jeu fait homme, fait à chaque instant ce qui est à faire, ce que demande et exige le jeu. Cela suppose une invention permanente, indispensable pour s'adapter à des situations indéfiniment variées, jamais parfaitement identiques. Cela n'assure pas l'obéissance mécanique à la règle explicite, codifiée (quand elle existe) [...]. Le sens du jeu n'est pas infaillible ; il est inégalement réparti, dans une société comme dans une équipe. Il est parfois en défaut [...]. Mais cette liberté d'invention, d'improvisation, qui permet de produire l'infinité des coups rendus possibles par le jeu (comme aux échecs) a les mêmes limites que le jeu ${ }^{65}$."

Si bien, qu'entre la détermination des règles du jeu et les stratégies mises en œuvre, il faut admettre une forme de magie sociale qui adapte les unes aux autres :

«Le sens pratique oriente des “choix” qui pour n'être pas délibérés n'en sont pas moins systématiques, et qui, sans être ordonnés et organisés par rapport à une fin, 
n'en sont pas moins porteurs d'une sorte de finalité rétrospective [...] [qui illustre] la rencontre quasi miraculeuse entre l'habitus et un champ, entre l'histoire incorporée et l'histoire objectivée, qui rend possible l'anticipation quasi parfaite de l'avenir inscrit dans toutes les configurations concrètes d'un espace de jeu ${ }^{66}{ }$.

Cette magie sociale est conceptuellement nécessaire pour réconcilier les contraires, apaiser les tensions : axiomatique, elle n'est jamais justifiée et explicitée. La cohérence du système ne pouvant dès lors que difficilement être mise en question, il faudrait plutôt en contester le caractère infalsifiable. Dès lors, faut-il jeter le bébé avec l'eau du bain? Comment se fait-il pourtant, une fois explicitées les difficultés internes et externes du modèle, que la séduction perdure, si l'on exclut l'hypothèse d'une malice diabolique chez le lecteur (et dont témoigne la diffusion dans l'historiographie d'un usage " spontané » de la notion de stratégie selon une conception voisine de celle de Bourdieu) ? Je soutiendrai plutôt que le programme ni-ni, cette tension entre deux enfers théoriques, avec toutes ses imperfections, signale une ligne problématique qui n'a pas épuisé ses effets de savoir. Problématique féconde lorsqu'elle s'efforce, dans une situation donnée complexe, de mettre au jour la relation entre les ressources sociales des agents, en particulier des individus et des groupes restreints, les règles du jeu à partir de ces ressources et le résultat final de la partie, moins éloquente peut-être, lorsqu'on passe à des collectivités plus larges (entreprises, institutions, nations, économies nationales ou régionales). Par ailleurs, les ambiguïtés, les tensions internes, la plasticité même du concept permettent à l'historien de l'utiliser de façon souple; ce qu'on perd en rigueur théorique, on le gagne en richesse d'analyse. Aussi la fécondité heuristique du programme ni-ni demeure-t-elle grande de par sa cohérence et sa capacité à poser des questions (si ce n'est à donner des réponses), qui, pour l'historiographie, ont le plus souvent été ignorées ou mal posées. Il convient en effet, à mon sens, plutôt que de juger abstraitement des bienfaits ou des méfaits de la théorie, d'envisager, plus simplement mais aussi plus stratégiquement, les coûts qu'elle induit et les gains qu'elle permet.

\section{NOTES}

1. Ce texte est la version abrégée d'une communication faite au séminaire «Choix et stratégies dans le développement économique et social », de P. Boutry, G. Delille et G. Levi, École française de Rome - École des Hautes Études en Sciences sociales, Rome, 24 janvier 1990.

2. J. Elster, "Le pire des mondes possibles. À propos de La Distinction de Pierre Bourdieu», Commentaire, 19, 1982, p. 445.

3. P. Bourdieu, La distinction. Critique sociale du jugement, Paris, Éd. de Minuit, 1979, p. 145. Les autres références aux travaux de P. Bourdieu figurent par la suite sans nom d'auteur.

4. La noblesse d'État, Paris, Éd. de Minuit, 19S9, p. 386.

5. Ibid.

6. Esquisse d'une théorie de la pratique, précédée de trois études d'ethnologie kabyle, Genève, Droz, 1972, p. 175 ; l'habitus est un «système de dispositions » produit de toute l'expérience biographique d'un individu, que l'on peut regrouper, bien qu'il n'y en ait pas deux identiques, en classes d'habitus, "sortes de programmes (au sens de l'informatique) historiquement montés ", qui 
donnent leur efficacité aux stimuli externes qui déclenchent l'action (Questions de sociologie, Paris, Éd. de Minuit, 1980, p. 75) ; la stratégie, c'est l'opus operatum, par rapport à l'habitus comme modus operandi (La noblesse d'État, op. cit., p. 389).

7. Questions de sociologie, op. cit., p. 119.

8. La noblesse d'État, op. cit., p. 386-387.

9. Questions de sociologie, op. cit., p. 119.

10. Les héritiers. Les étudiants et la culture (avec J.-C. Passeron), Paris, Éd. de Minuit, 1964 ; Un art moyen. Essai sur les usages sociaux de la photographie (avec L. Boltanski, R. Castel, J.C. Chamboredon), Paris, Éd. de Minuit, 1965 ; L'amour de l'art. Les musées d'art européens et leur public (avec A. Darbel), Paris, Éd. de Minuit, 1969; La reproduction. Éléments pour une théorie du système d'enseignement (avec J.-C. Passeron), Paris, Éd. de Minuit, 1970; Ce que parler veut dire. L'économie des échanges linguistiques, Paris, Fayard, 1982.

11. Esquisse d'une théorie de la pratique, op. cit.

12. Choses dites, Paris, Éd. de Minuit, 1987.

13. Homo academicus, Paris, Éd. de Minuit, 1984.

14. Esquisse d'une théorie de la pratique, op. cit.; Le sens pratique, Paris, Éd. de Minuit, 1980 ; Homo academicus, op. cit. ; Choses dites, op. cit.

15. La distinction, op. cit.; La noblesse, op. cit.

16. Esquisse d'une théorie de la pratique, op. cit.

17. Le sens pratique, op. cit.

18. La distinction, op. cit.

19. La noblesse, op. cit.

20. Le sens pratique, op. cit., p. 270.

21. Choses dites, op. cit., p. 80.

22. Ibid., p. 127.

23. «La théorie de l'habitus vise à fonder la possibilité d'une science des pratiques échappant à l'alternative du finalisme et du mécanisme ", Questions de sociologie, op. cit., p. 119. Le programme ni-ni est explicitement formulé in Le sens pratique, op. cit., p. 51-87.

24. Le sens pratique, op. cit., p. 85-86.

25. Questions de sociologie, op. cit., p. 116.

26. Cette critique est d'ailleurs commune aux sociologues, tels M.Crozier et R. Boudon, reprenant, dans des logiques différentes, le paradigme économique. Cf. A. Caillé, «La sociologie de l'intérêt est-elle intéressante? (à propos de l'utilisation du paradigme économique en sociologie) », Revue française de sociologie, XXII, 1981, p. 257-274.

27. "Espace social et genèse des "classes" ", Actes de la recherche en sciences sociales, 52-53, 1984, p. 3.

28. Questions de sociologie, op. cit., p. 58-59.

29. J. Rancière, «L'éthique de la sociologie », in Collectif «Révoltes logiques", L'empire du sociologue, Paris, La Découverte, 1984.

30. Questions de sociologie, op. cit., p. 76.

31. "L'illusion biographique ", Actes de la recherche en sciences sociales, 62-63, 1986, p. 71.

32. Choses dites, op. cit., p. 129.

33. G. Levi, «Les usages de la biographie », Annales ESC, XLIV, 1989, p. 1325-1336.

34. M. J. Piore, C. Sabel, Les chemins de la prospérité, Paris, Hachette, 1989.

35. J. Rancière, op. cit., p. 30-32.

36. Le sens pratique, op. cit., p. 180.

37. Questions de sociologie, op. cit., p. 10.

38. Le sens pratique, op. cit., p. 186.

39. J. Elster, op. cit., p. 445. 
40. F. Bon, Y. Schemeil, «La rationalisation de l'inconduite : comprendre le statut du politique chez Pierre Bourdieu », Revue française de science politique, XXX, 1980, p. 1198-1228.

41. «Espace social », op. cit., p. 5.

42. C. Babbage, On the Economy of Machinery and Manufactures, Londres, 1835 ; A. Ure, The Philosophy of Manufactures, Or an Exposition of the Scientific, Moral and Commercial Economy of the Factory System of Great Britain, Londres, 1835; cf. S. Marglin, «Origines et fonctions de la parcellisation des tâches. À quoi servent les patrons? ", in A. Gorz, ed., Critique de la division du travail, Paris, Seuil, 1973, p. 41-89.

43. Choses dites, op. cit., p. 124.

44. Le sens pratique, op. cit., p. $85 \mathrm{n}$.

45. Questions de sociologie, op. cit., p. 119.

46. Choses dites, op. cit., p. 125.

47. L. J. D. Wacquant, C. Jackson Calhoun, «Intérêt, rationalité et culture. À propos d'un récent débat sur la théorie de l'action ", Actes de la recherche en sciences sociales, 78, 1989, p. 41-60.

48. "La production de la croyance. Contribution à une économie des biens symboliques", Actes de la recherche en sciences sociales, 13, 1977, p. 9.

49. J. Elster, op. cit., p. 449.

50. Le sens pratique, op. cit., p. 104-105.

51. D. Memmi, «Le désintéressement ", intervention au séminaire du Centre de sociologie de l'Éthique, EHESS-CNRS, juin 1989.

52. A. Caillé, op. cit., p. 258 ; A. L. Cot, B. Lautier, « Métaphore économique et magie sociale chez Pierre Bourdieu », in Collectif « Révoltes logiques », op. cit., p. 70-85.

53. Choses dites, op. cit., p. 124 ; ou: «Rompre avec l'économisme pour décrire l'univers des économies possibles, c'est échapper à l'alternative de l'intérêt purement matériel, étroitement économique, et du désintéressement et se donner le moyen de satisfaire au principe de raison suffisante qui veut qu'il n'y ait pas d'action sans raison d'être, c'est-à-dire sans intérêt ou, si l'on préfère, sans investissement dans un jeu et un enjeu, illusion, commitment ", Le sens pratique, op. cit., p. $85 \mathrm{n}$.

54. À l'exception de: P. Bourdieu, M. de Saint-Martin, "Le patronat", Actes de la recherche en sciences sociales, 21-22, 1978 ; les études de cas consacrées au "champ économique " sont peu représentées dans les Actes de la recherche en sciences sociales.

55. K. Polanyi, The Great Transformation. The Political and Economical Origins of our Time, New York, Holt, Rinehart \& Winston, 1944, trad. française, Paris, Gallimard, 1983; K. Polanyi, C. M. Arensberg, H. W. Pearson, eds, Trade and Markct in the Early Empires, Chicago, Gateway, 1971.

56. A. Hirschman, The Passions and the Interests. Political Arguments for Capitalism before its Triumph, Princeton, Princeton University Press, 1977, trad. française, Paris, Presses universitaires de France, 1980.

57. L. Boltanski, L. Thévenot, Les économies de la grandeur, Paris, Presses universitaires de France, 1987 ; Les conventions économiques, Cahiers du centre d'études de l'emploi, Paris, Presses universitaires de France, 1985 ; R. Salais, L. Thévenot, eds, Le travail. Marchés, règles, conventions, Paris, INSEE-Economica, 1986.

58. Comment en effet mesurer la valeur d'échange et de conversion d'un capital à un autre, J. Rancière, op. cit., p. 31.

59. Choses dites, op. cit., p. 130.

60. C. Lévi-Strauss, " Histoire et ethnologie », Annales ESC, XXXVIII, 1983, p. 1229-1230.

61. "Je ne me reconnais pas dans ce que Lévi-Strauss a dit récemment à propos des recherches sur ce qu'il appelle les “sociétés à maisons". Bien que je ne puisse pas ne pas me sentir concerné, puisque j'ai contribué à réintroduire dans la discussion théorique en ethnologie une de ces sociétés où les actes d'échange, matrimoniaux ou autres, semblent avoir pour « sujet » la maison, 
la maysou, l'oustau; et aussi à formuler la théorie du mariage comme stratégie. » (Choses dites, op. cit., p. 77).

62. Ibid., p. 79.

63. É. Durkheim, De la division du travail social [1893], Paris, Presses universitaires de France, 1986, p. 369 ; à comparer avec: "À un volume déterminé de capital hérité, correspond un faisceau de trajectoires à peu près équiprobables conduisant à des positions à peu près équivalentes - c'est le champ des possibles objectivement offert à un agent déterminé », La distinction, op. cit., p. 122.

64. "Espace social », op. cit., p. 5.

65. Choses dites, op. cit., p. 79.

66. Le sens pratique, op. cit., p. 111.

\section{RÉSUMÉS}

Cette note de lecture vise à déployer l'espace de la notion de «stratégie » construite dans les textes de Pierre Bourdieu. Analysée dans ses relations avec les notions connexes d'habitus et de capital, la "stratégie » est l'expression d'un programme ni-ni, entre subjectivisme et objectivisme, entre rationalité des acteurs et structure. Elle est ainsi contrainte, tant du côté du sujet que du mode, de la fin et de l'historicité de l'action, par des tensions qui, loin de la rendre caduque, rendent son emploi par les historiens d'autant plus fécond.

This note aims to elucidate the notion of "strategy" constructed in the works of Pierre Bourdieu. Analysed in its relationships with the allied notions of «habitus " and of capital, «strategy » is the expression of a neither-nor programme, between subjectivism and objectivism, between rationality of actors and structure. It is thus restrained as much on the side of the subject as on the side of custom, goal and historicity of action by tensions, which far from making it decrepit, render its use by historians all the more fruitful. 